Article

\title{
Rapid and Efficient Estimation of Pea Resistance to the Soil-Borne Pathogen Fusarium oxysporum by Infrared Imaging
}

\author{
Nicolas Rispail * and Diego Rubiales \\ Institute for Sustainable Agriculture, CSIC, Alameda del Obispo s/n, Apdo. 4084, Córdoba 14080, \\ Spain; E-Mail: diego.rubiales@ias.csic.es \\ * Author to whom correspondence should be addressed; E-Mail: nrispail@ias.csic.es; \\ Tel.: +34-957-499-211; Fax: +34-957-499-252.
}

Academic Editor: Stephane Evoy

Received: 12 November 2014 / Accepted: 30 January 2015 / Published: 9 February 2015

\begin{abstract}
Fusarium wilts are widespread diseases affecting most agricultural crops. In absence of efficient alternatives, sowing resistant cultivars is the preferred approach to control this disease. However, actual resistance sources are often overcome by new pathogenic races, forcing breeders to continuously search for novel resistance sources. Selection of resistant accessions, mainly based on the evaluation of symptoms at timely intervals, is highly time-consuming. Thus, we tested the potential of an infra-red imaging system in plant breeding to speed up this process. For this, we monitored the changes in surface leaf temperature upon infection by $F$. oxysporum f. sp. pisi in several pea accessions with contrasting response to Fusarium wilt under a controlled environment. Using a portable infra-red imaging system we detected a significant temperature increase of at least $0.5{ }^{\circ} \mathrm{C}$ after 10 days post-inoculation in the susceptible accessions, while the resistant accession temperature remained at control level. The increase in leaf temperature at 10 days post-inoculation was positively correlated with the AUDPC calculated over a 30 days period. Thus, this approach allowed the early discrimination between resistant and susceptible accessions. As such, applying infra-red imaging system in breeding for Fusarium wilt resistance would contribute to considerably shorten the process of selection of novel resistant sources.
\end{abstract}

Keywords: infra-red imaging system; Fusarium oxysporum; Fusarium wilt; leaf temperature; Pisum sativum; screening for resistance; plant breeding 


\section{Introduction}

Fusarium wilts are widespread diseases caused by many forms of the soil-borne pathogen Fusarium oxysporum, affecting many agricultural crops, including most legumes, cucurbits, tomato, strawberry, cotton and banana [1,2]. This soil-borne pathogen can survive as thick-walled chlamydospores, which remain viable in the soil for many years, which makes its control difficult. Upon host recognition, the germinating fungus is able to penetrate the root and reach the vascular vessels where it grows profusely leading to a rapid plant death, in part due to drastic water stress [3]. Once established, this pathogen is very difficult to eradicate since it can grow in the absence of a compatible host.

In absence of alternative efficient or economically viable methods of control, the use of resistant cultivars is the most used approach to control Fusarium wilt. However sources of resistance are somewhat limited and single genes, which have been identified and used in breeding, are rapidly overcome by new races of the pathogen [4]. It is thus essential to continuously search for novel sources of resistance to complement and reinforce the actual resistance of elite cultivars. This search requires the availability of large germplasm collections and of precise and accurate screening techniques [4]. Efficient screening methods have been established and described for many crops [4-8] but these are highly time-consuming. Indeed, symptoms usually initiate on oldest leaves around 7-10 days post-inoculation (dpi) following artificial inoculation in growth room and they will progress through the plant until complete plant death that usually occurs around 20 to 30 dpi in susceptible genotypes. Late wilting or moderately resistant genotypes may die even later. Thus the whole process requires more than one month to be completed under controlled environments. In field conditions with natural inoculation an accurate evaluation would require much more time. Alternative disease evaluation methods accelerating the screening and selection of resistant lines would thus be advantageous.

The recent development of imaging-based phenotyping such as the evaluation of surface temperature by infra-red imaging system and measurement of chlorophyll fluorescence is revolutionizing agriculture and plant science. Infra-red thermal imaging is a non-contact, non-destructive and rapid technique which provides a temperature map of the targeted material or plant. In addition, the infra-red camera is easy to handle and highly accurate making it suitable for both laboratory and field studies [9]. In general, plant surface temperature is dependent on transpiration rate. At high transpiration rate, the leaf temperature is cooling while at lower transpiration rate, this temperature increases. Surface leaf temperature is thus an indirect parameter to evaluate the overall physiological status of a plant [10]. As such, it has been largely applied to monitor plant water status [11]. It is also powerful to identify stressed plants by both biotic and abiotic stresses [12]. Thus, this method has been applied to detect the presence of several plant diseases both in field and laboratory indicating that infra-red imaging could be used to detect vascular wilt diseased plants [10,13-16]. Application of thermal imaging or thermography approaches offers great potential for breeding [17]. For instance, this approach was successfully used to screen for barley and Arabidopsis mutants deficient in stomatal movements [18,19] and to identify drought or salinity tolerant genotypes in rice [20], barley [21], wheat [22] and maize [23]. However, the potential of these methods in breeding for disease resistance has not been explored yet. Thus, we used the pea- $F$. oxysporum pathosystem as a model to evaluate the potential of infra-red thermal imaging as an indirect screening method to discriminate susceptible and resistant plants speeding up the evaluation of Fusarium wilt resistance in crops and the selection of resistant accessions. 


\section{Results}

Prior to the infra-red analysis of the accessions, we evaluated their responses to the disease from 7 to $30 \mathrm{dpi}$ in order to estimate the area under the disease progression curve (AUDPC) for further analysis and to confirm their resistance status. As expected, we detected continuity in the disease response of the different accessions from resistant to susceptible (Figure 1). The disease response of the accessions was broadly similar to that previously reported [5]. Significant differences were detected between accessions $(p<0.001)$ and mean comparison by Duncan multiple range test allowed the discrimination between the resistant accessions JI 1412, JI 1747, JI 2480 and New Season from the moderately susceptible accessions JI 502, JI 2302 and Little Marvel while the last accession JI 1213 was classified as susceptible (Figure 1).

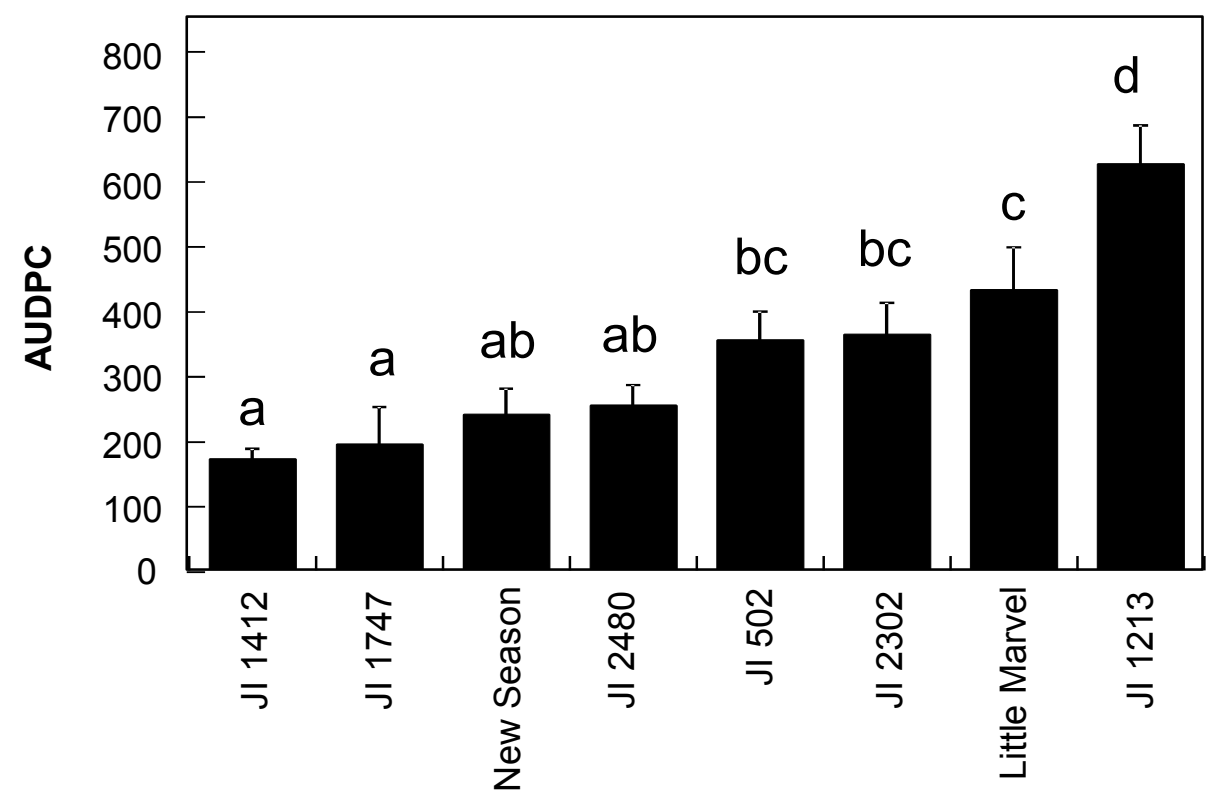

Accessions

Figure 1. Assessment of Fusarium oxysporum f. sp. pisi (Fop) disease symptoms in the selected Pisum sativum accessions. The histogram shows the area under the disease progression curve (AUDPC) calculated for each P. sativum accession from the periodic evaluation of Fop disease symptoms expressed as percentage of wilting leaves per plant. Vertical bars are standard errors for $\mathrm{n}=10$. Different letters between each histogram indicates significant difference between values according to Duncan multiple range test at $\alpha=0.05$.

Comparison of the surface leaf temperature between control and inoculated plants showed an early and slight reduction of temperature in all accessions following inoculation (Figure 2). Thereafter, temperature oscillated in most accessions during the first $6 \mathrm{dpi}$, in most cases following a similar trend and probably reflecting plant adaptation to the uneven stresses applied at inoculation time. After that initial period, the leaf temperature of inoculated resistant accessions stabilized to control level while that of moderately and highly susceptible accessions increased more than $0.5{ }^{\circ} \mathrm{C}$ (Figure 2). 


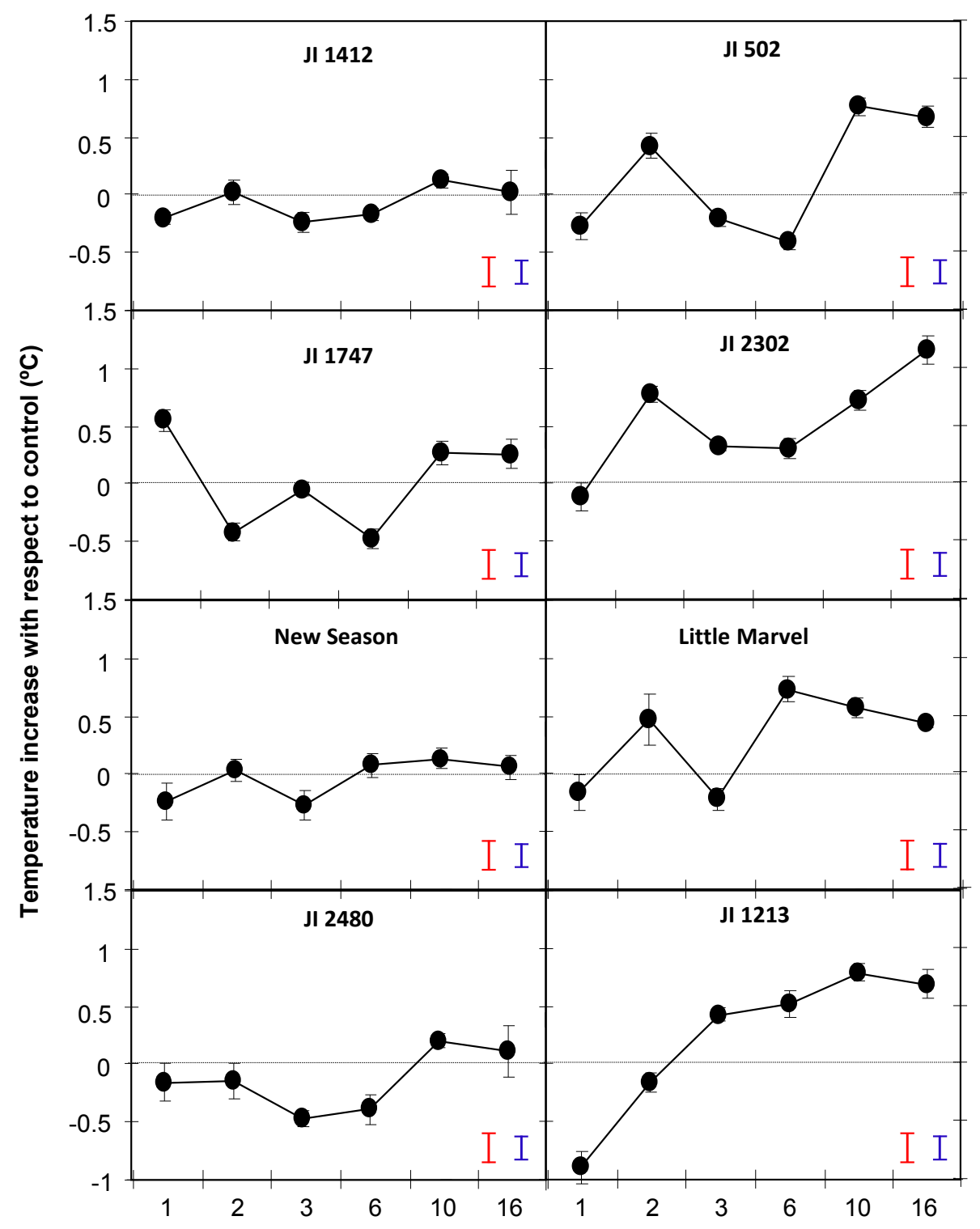

Figure 2. Evolution of the surface leaf temperature of $P$. sativum plants following inoculation with $F$. oxysporum f. sp. pisi (Fop). The graphs represent the evolution of the differences in surface temperature between Fop-inoculated and control plants for the resistant accessions JI 1412, JI 1747, JI 2480 and New Season, the partially resistant, JI 502, JI 2306 and Little Marvel, and the susceptible accession JI 1213 according to the time post-inoculation. Surface leaf temperatures were measured by infra-red imaging system. Vertical bars on each data point are standard errors for $n=10$. Red and blue vertical bars represent the LSD values at $\alpha=0.05$ between genotypes ( $\mathrm{LSD}=0.26)$ and time points $(\mathrm{LSD}=0.21)$, respectively.

Thus by 10 dpi the temperature of JI 1412, JI 1747, JI 2480 and New Season was not different from that of control, non-inoculated, plants (Figure 2). This was also detectable by visual observation of the snapshots obtained with the camera (Figure 3). By contrast moderately and highly susceptible plants increased its leaf temperature between 0.6 and $0.85{ }^{\circ} \mathrm{C}$ in respect to their non-inoculated controls (Figure 2). This slight increase of temperature was also appreciated visually in the images (Figure 3 ). 


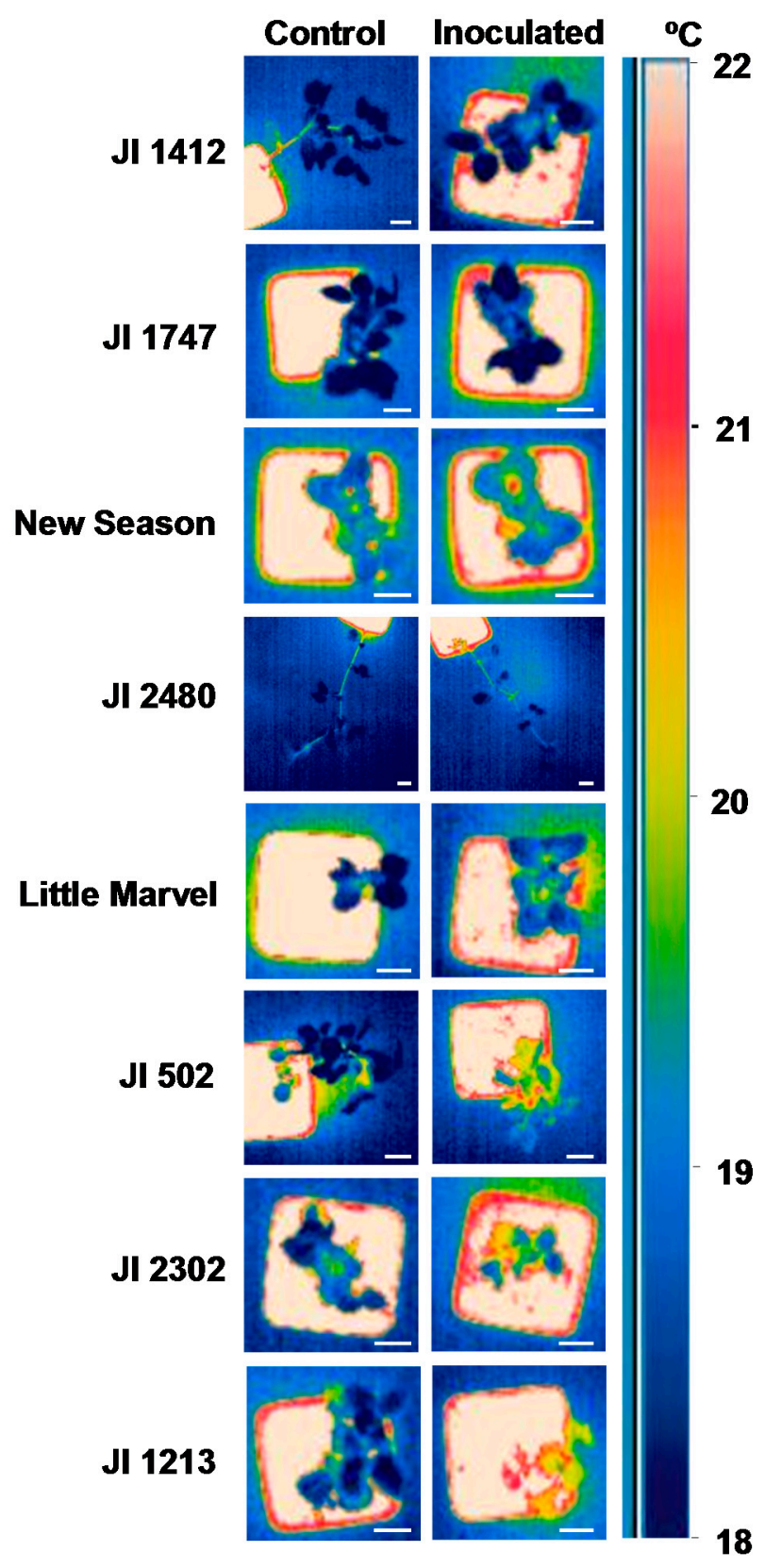

Figure 3. Comparison of the surface leaf temperature between control and inoculated $P$. sativum plants with $F$. oxysporum f. sp. pisi (Fop) at 10 days post-inoculation. The figure shows a representative image of each accession maintained non-inoculated (Control, Left) and inoculated with Fop (Right). Scale bars indicate $2 \mathrm{~cm}$.

In order to determine whether the temperature increases and the susceptible/resistance responses were statistically correlated, a Spearman rank correlation analysis was performed (Table 1). As expected the disease rating at 10 or $16 \mathrm{dpi}$ and the AUDPC values were highly correlated among them. Interestingly, Spearman rank correlation also indicated a positive and significant correlation between temperature increases at $10 \mathrm{dpi}$ and the disease symptoms either recorded as disease ratings at $10(0.551, p<0.001)$ or 16 dpi $(0.628, p<0.001)$ or as the AUDPC value $(0.658, p<0.001)$. A significant correlation was also detected between temperature increases at $16 \mathrm{dpi}$ and disease ratings or AUDPC. However at this time the correlation coefficient was smaller with a mean of 0.396 . Further, when plotting the changes in 
leaf temperature at 10 dpi against the AUDPC values two groups were also clearly discriminated, one of them comprising the resistant accessions and the other clustering the moderately and highly susceptible accessions (Figure 4). The regression analysis showed that data fitted $\left(\mathrm{R}^{2}=0.694\right)$ a linear regression curve with a mean difference of temperature of $0.5^{\circ} \mathrm{C}$ between the resistant and susceptible genotype groups.

Table 1. Spearman rank correlation coefficient for disease symptom levels and changes in surface leaf temperature comparisons for $\mathrm{n}=10$.

\begin{tabular}{cccccc}
\hline & $\begin{array}{c}\text { Disease } \\
\text { Rating 10 dpi }\end{array}$ & $\begin{array}{c}\text { Disease } \\
\text { Rating 16 dpi }\end{array}$ & $\begin{array}{c}\text { AUDPC } \\
\text { Value }\end{array}$ & $\begin{array}{c}\mathbf{T}^{\circ} \text { Increase } \\
\text { at 10 dpi }\end{array}$ & $\begin{array}{c}\mathbf{T}^{\circ} \text { Increase at } \\
\mathbf{1 6 ~ d p i}\end{array}$ \\
\hline Disease rating 10 dpi & 1.000 & - & - & - & - \\
Disease rating 16 dpi & $0.631 * * *$ & 1.000 & - & - & - \\
AUDPC value & $0.902 * * *$ & $0.891 * * *$ & 1.000 & - & - \\
$\mathrm{T}^{\circ}$ increase 10 dpi & $0.540 * * *$ & $0.628 * * *$ & $0.658 * * *$ & 1.000 & - \\
$\mathrm{T}^{\circ}$ increase 16 dpi & $0.321 * *$ & $0.442 * * *$ & $0.424 * * *$ & $0.380 * *$ & 1.000 \\
\hline$* *$ and $* * *$ indicates the significance of the correlation coefficient according to the Student $t$ test at 0.01 and \\
0.001 level respectively.
\end{tabular}

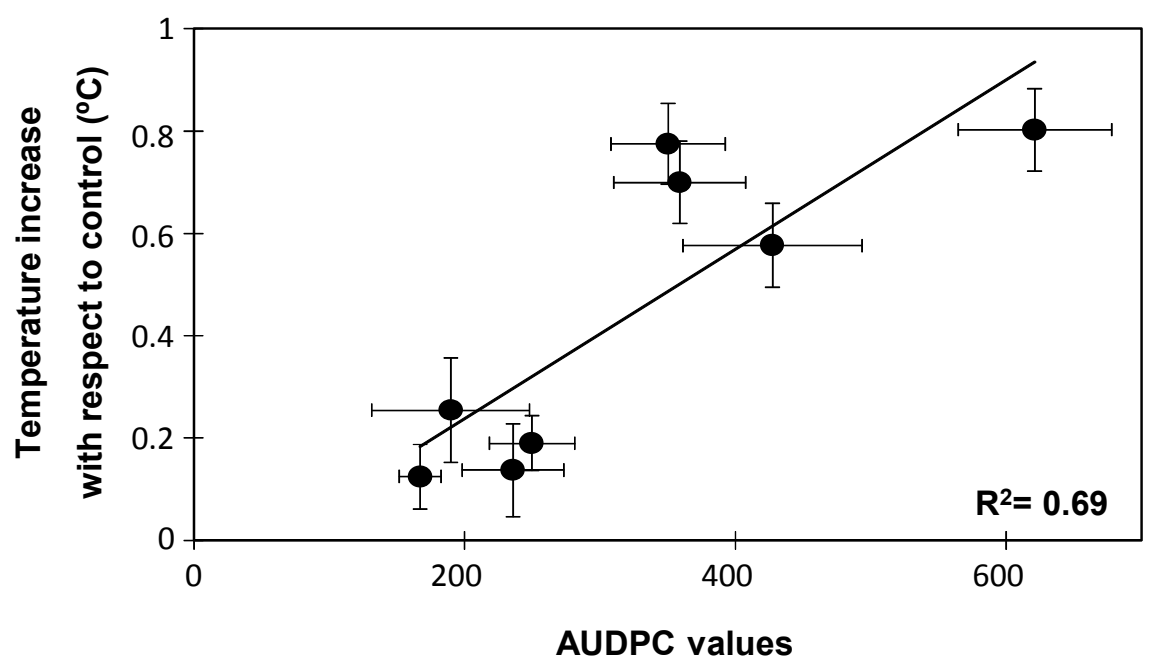

Figure 4. Relationship between the level of susceptibility to F. oxysporum f. sp. pisi (Fop) and surface leaf temperature. The graphic represents the linear correlation calculated for the differences in surface leaf temperature detected for each $P$. sativum accession between inoculated and control plants at 10 days post-inoculation with Fop in relation with their corresponding AUDPC values. Horizontal bars are standard errors for AUDPC values with $\mathrm{n}=10$ while vertical bars are standard errors for the differences in surface leaf temperature calculated with $\mathrm{n}=10$.

\section{Discussion}

Infra-red thermography is a very powerful method to monitor changes in plant water status in vivo. Thus it is being largely used in agriculture and plant science for a wide range of applications, from scheduling irrigation to evaluating fruit maturity $[9,24]$. However, the usefulness of thermal imaging has not been sufficiently explored in disease resistance breeding. Here, we show that infra-red thermography 
allows the detection of pea plants infected by the vascular wilt pathogen $F$. oxysporum and the discrimination between susceptible and resistant pea cultivars at a very early stage of the interaction. As such infra-red thermography might be very useful for future screening for vascular wilt resistance in germplasm collections of pea and other plant species.

Surface leaf temperature is strongly correlated with the plant transpiration rate. Thus, during water stress-induced stomata closure the transpiration rate decreased and as a result, the surface leaf temperature increased compared to well-watered control plants [11,25]. A rapid closure of stomata has also been detected in response to infection by air-borne pathogens leading to an increase in surface leaf temperature $[10,26]$. This has led to the application of remote sensing approaches to detect foliar diseases in the field $[13,15]$. Interestingly, in plants infected by fungal root pathogens, such as $F$. solani, Rhizoctonia solani, Verticillium dahliae, a higher surface temperature with respect to healthy plants has been observed $[13,27]$. Changes in stomatal conductance, transpiration rate and superficial leaf temperature in susceptible wilted plants upon infection by $F$. oxysporum have also been reported [16,28,29]. Accordingly, we detected an increase in the leaf temperature of susceptible accessions of pea upon infection with the soil-borne pathogen $F$. oxysporum (Figures 2 and 3). This reinforced the close relationship previously established between vascular wilt and drought $[28,30]$.

From a mechanistic point of view several processes might account for the temperature increase in the susceptible accessions. It is often believed that the wilting and death of $F$. oxysporum-infected plants are due to perturbation of the water fluxes within the plants $[28,30]$. The increase in leaf temperature and associated stomatal closure might be related to the vessel plugging induced by the intensive fungal growth within xylem vessels and by the attempt of plant defence that blocked xylem cells $[16,30]$. However, recent studies showed that the water flux while disturbed was not blocked by these defence responses [28]. In accordance with this, we did not observe significant changes of the surface leaf temperature in the resistant pea accessions (JI 1412, JI 1747, JI 2480 and New Season) upon infection. Alternatively the temperature increase observed in the susceptible plants might be related to toxin production [28,29,31]. Fusaric acid has been shown to be one of the main toxins produced by F. oxysporum [32] and an important pathogenicity factor since it induces wilting symptoms in several plant species, including cucumber, banana and pepper [28,29,31-33]. Several studies in banana, cucumber and watermelon showed that application of fusaric acid to the root led to a rapid ABA-dependent stomatal closure. As such, the application of fusaric acid mimicked the temperature rise of the leaf detected upon infection with the pathogen in these species [28,29,31]. Independently of the causal effect leading to stomatal closure and transpiration rates, this temperature increase was only detected in susceptible accessions since the surface leaf temperature of resistant pea accessions was maintained at control level (Figure 2). Thus by monitoring the changes in leaf temperature, we were also able to differentiate the response observed between the resistance and susceptible accessions at an early stage of the interaction which opens the possibility to use infra-red thermography in Fusarium wilt resistance breeding.

The genotype-dependent oscillations of temperature observed in most accessions during the first 6 dpi probably reflected the plant adaptation to the inoculation process and the attempt of plant defence. Thereafter, the surface leaf temperature rise of about $0.5{ }^{\circ} \mathrm{C}$ for all susceptible accessions while it remained at control level in the resistant accessions (Figure 2). In addition, the changes in leaf temperature measured at either 10 or 16 dpi were positively correlated with the disease symptom rates measured at 16 dpi and by the AUDPC (Table 1 and Figure 4). Surprisingly, the correlation between the 
disease parameters and the leaf temperature changes was higher when the surface temperature was measured at $10 \mathrm{dpi}$ than at $16 \mathrm{dpi}$. Although disease symptoms were only initiating at $10 \mathrm{dpi}$, a previous study showed that the fungus had already colonized the whole plant of susceptible pea accession at this stage [5]. Thus at this stage, the temperature difference might reflect the pathogen action within susceptible plants. By contrast, in the resistant accessions, the pathogen only colonized the root system being efficiently blocked at crown level by the establishment of defence reactions [5]. This might explain that the leaf temperature of inoculated resistant accessions rally control level by 10 dpi (Figure 2). At days 16 , disease symptoms are clearly visible on all susceptible plants and on some older leaves of some resistant accessions. The presence of dying leaves on these plants may distort the leaf temperature of the plant which might explain the lower correlation observed between disease symptom and temperature at this later stage. Altogether this suggests that the measurement of leaf temperature at 10 dpi better reflect the plant physiological response to the pathogen.

Although the changes in the temperature between accessions following pathogen attack were relatively small, it allowed separating the accessions in two groups according to their level of susceptibility before the appearance of the initial visual symptoms (Figures 2 and 3 ) and hence, it might be very valuable to identify and select resistant accessions. From a breeding perspective, while several methods of inoculation have been developed to allow rapid and reproducible Fusarium wilt [5,34], the identification of resistant accessions to this disease is mainly based on the evaluation of symptoms at timely intervals which is time-consuming [4,5]. The application of thermal imaging system to monitor changes in leaf temperature at an early stage of the interaction may contribute to reduce the time needed for the identification and selection of resistant pea plants to Fusarium wilt.

Screenings for resistance to soil-borne diseases are performed either under field condition or under a controlled environment. While field testing is often considered a more appropriate approach, the uneven distribution of pathogenic population in the soil and the presence of additional soil pathogen may interfere with the results [4]. To circumvent these drawbacks, screening approaches under controlled environments have been developed and shown to be well-correlated with field testing. Although, the limited space is often an important issue, this approach allows for large-scale screening of germplasm collections $[4,5,8]$. Surface temperature is strongly affected by environmental conditions thus this method may not be adequate for field screening which are subject to very variable environmental conditions. However, implementation of infra-red thermography may be very useful to speed up the screening of large germplasm collections under controlled environments in which plants are maintained in more stable and constant conditions. This is supported by the fact that resistant accessions were clearly separated from the susceptible ones when comparing the image thermography of control and inoculated plants at 10 and to a lesser extent at 16 dpi (Figure 3). The significant and positive correlation between the AUDPC values and the changes in leaf temperature at $10 \mathrm{dpi}$ also support that leaf surface temperature at this time can be used as indirect parameter for selection under controlled environment. Although detailed evaluation of disease symptoms might be more accurate, it is highly time-consuming. Detailed analyses of infra-red thermography images and calculation of the overall surface temperature of all plants is also time-consuming. However, visual monitoring of the infra-red thermography images was found sufficient to discriminate between resistant and susceptible accessions as shown in Figure 3 and is a very rapid approach since it can be done during image acquisition without further image 
processing. As a result, the application of infra-red thermography may reduce the time needed to screen large germplasm collections.

Altogether, our results support the usefulness of thermal imaging system to screen large pea germplasm collections for $F$. oxysporum disease resistance or to select resistant individuals within large segregating populations under controlled environment. Changes in temperature or transpiration rate have been detected in susceptible accessions of many plant species in response not only to Fusarium wilt disease but also many other air-borne and soil-borne diseases [16,28,34-36]. Such changes in stomatal conductance or transpiration rate have not been detected or only transiently in resistant accessions (Figure 2) [36,37] suggesting that this can be an interesting parameter for screening for resistance not only for Fusarium wilt but also to other important diseases such as powdery mildew, rust or root rots.

\section{Experimental Section}

\subsection{Fungal Isolate and Culture Conditions}

The Fusarium oxysporum f. sp. pisi (Fop) race 2 strain R2F42 was used in all the experiments. The fungal strain was stored as microconidial suspensions at $-80{ }^{\circ} \mathrm{C}$ in $30 \%$ glycerol. For microconidia production, cultures were grown in potato dextrose broth (PDB; Difco, Detroit, MI, USA) at $28{ }^{\circ} \mathrm{C}$ in a shake culture set at $170 \mathrm{rpm}$.

\subsection{Plant Material and Growth Conditions}

Eight accessions of P. sativum (JI 502, JI 1213, JI 1412, JI 1747, JI 2302, JI 2480, Little Marvel and New season) with contrasting responses to Fop were used in this study [5]. Pea seeds were surface-sterilized for $20 \mathrm{~min}$ in a $20 \%$ solution of sodium hypochlorite and then rinsed three times with sterile water before being wrapped in wet filter paper in a Petri dish. Then the seeds were stratified for two days at $4{ }^{\circ} \mathrm{C}$ in the dark and incubated at $20 \pm 2{ }^{\circ} \mathrm{C}$ until germination. Once germinated, the seedlings were transferred to pots $\left(36 \mathrm{~cm}^{2} \times 8 \mathrm{~cm}\right)$ containing sterile vermiculite $(2-8 \mathrm{~mm}$ diameter $)$ and grown in a controlled environmental chamber under a $16 / 8 \mathrm{~h}$ light-dark photoperiod at $26 \pm 2{ }^{\circ} \mathrm{C}$ temperature regime with $200 \mu \mathrm{mol} \cdot \mathrm{m}^{-2} \cdot \mathrm{s}^{-1}$ of illumination. Plants were watered every three days with tap water.

\subsection{Inoculation and Disease Assessment}

Seven-day-old pea seedlings (2-3 node stage) were inoculated following the root dipping method previously described [5]. For this, each plant were uprooted, cleaned off vermiculite and the whole root system was submerged for $30 \mathrm{~min}$ in a solution of $5 \times 10^{6} \mathrm{Fop}$ microconidia $\cdot \mathrm{mL}^{-1}$ (inoculated plants) or in sterile water (control plants). Seedlings were then planted in individual pots containing sterile vermiculite and placed back in the growth chamber with the same growth condition as indicated above. 10 plants per accession were used for each treatment and organized in two blocks of 5 plants per accession. In each block, inoculated plants were maintained in a tray near the control plants. In addition, blocks were shuffled around the growth chamber every three days to homogenize the growth condition between blocks. Fop symptoms were rated every three days from 7 th to 30th dpi by estimating the percentage of leaves with symptoms per plant. At the end of the experiment, these data were used to calculate the AUDPC value as described previously [5]. 


\subsection{Thermal Imaging of Control and Inoculated Plants}

Infra-red images of pea plants were obtained with a Thermovision A40M (FLIR, Wilsonville, OR, USA) thermal camera equipped with a $43^{\circ}$ FOV lens and connected to computer via the IEEE-1394 protocol. The image sensor was a Focal Plane Array (FPA) based on uncooled microbolometers with a resolution of $320 \times 240$ pixels, a spectral response in the range $7.5-13 \mu \mathrm{m}$, with $0.08{ }^{\circ} \mathrm{C}$ sensitivity at $30{ }^{\circ} \mathrm{C}$ and $0.1 \mathrm{~mm}$ minimal focus distance. Digital thermograms were acquired with the temperature range set between +10 and $+55^{\circ} \mathrm{C}$ with the spectral rainbow color scheme and the autoadjust function in off with FSCAP software (FLIR). The leaf temperature for each plant was determined by calculating the mean temperature of four spots $(3 \mathrm{~mm}$ diameter) placed on four distinct leaves. Leaf temperature was monitored at 1, 2, 3, 6, 10 and 16 dpi.

\subsection{Statistical Analysis}

Differences in surface leaf temperature and in disease severity between accessions were statistically assessed by ANOVA using contrast analysis (Scheffe) and Duncan multiple range test respectively. Spearman rank correlation test were performed to determine correlations between changes in superficial leaf temperature. All statistical analyses were performed with SPSS Statistics v. 22 software (IBM Corp., Armonk, NY, USA).

\section{Conclusions}

In this study, the possibility to use infra-red thermography for disease resistance breeding was evaluated. Using the pea- $F$. oxysporum pathosystem, we demonstrated that infra-red thermography can not only indicate the presence of this soil-borne pathogen within susceptible pea plants but also discriminate between resistant and susceptible plants at an early stage of the interaction under controlled environment. In this pathosystem, a significant increase of the superficial leaf temperature was detected as early as $10 \mathrm{dpi}$ for the susceptible accessions while the temperature of resistant accessions rallied control level. The temperature increase in susceptible accessions was positively correlated with the disease symptoms estimated over a period of 30 days after inoculation. Thus the implementation of infra-red thermography in breeding for resistance to Fusarium wilt may reduce the time required to identify and select resistant plants while screening large germplasm pea collections under controlled environment. Since infection by other plant pathogens also led to an increase of temperature in susceptible plants from different species, the usefulness of infra-red thermography in resistance breeding may be expanded to additional diseases and crops.

\section{Acknowledgments}

This work was supported by the European KBBE project ABSTRESS (FP7-KBBE-2011-5-289562) and the AGL2011-22524 of the Spanish Ministry of Science and Innovation and co-financed by European fund for regional development (FEDER). Nicolas Rispail is holder of a Ramón y Cajal postdoctoral position from the Spanish Ministry of Economy and Competitiveness. We thank Weidong Chen (USDA-ARS, Pullman, Washington, DC, USA) for providing the fungal isolate and the Genetic 
Resource Unit of the John Innes Centre (Norwich, UK) for providing the JI accessions of pea used in this study. We also thank Pablo J. Zarco-Tejada for technical advices with the infra-red imaging system.

\section{Author Contributions}

Nicolas Rispail designed, performed the experiments, interpreted the data and wrote the manuscript; Diego Rubiales participated in the design of the experiments, supervised the experiments and contributed in drafting and revising the manuscript. All authors read and approved the final manuscript.

\section{Conflicts of Interest}

The authors declare no conflict of interest.

\section{References}

1. Gordon, T.R.; Martyn, R.D. The evolutionary biology of Fusarium oxysporum. Ann. Rev. Phytopathol. 1997, 35, 111-128.

2. Rubiales, D.; Fondevilla, S.; Chen, W.; Gentzbittel, L.; Higgins, T.J.V.; Castillejo, M.A.; Singh, K.B.; Rispail, N. Achievements and challenges in legume breeding for pest and disease resistance. Crit. Rev. Plant Sci. 2015, 34, 195-236.

3. Di Pietro, A.; Madrid, M.P.; Caracuel, Z.; Delgado-Jarana, J.; Roncero, M.I.G. Fusarium oxysporum: Exploring the molecular arsenal of a vascular wilt fungus. Mol. Plant Pathol. 2003, 4, 315-325.

4. Infantino, A.; Kharrat, M.; Riccioni, L.; Coyne, C.J.; McPhee, K.E.; Grunwald, N.J. Screening techniques and sources of resistance to root diseases in cool season food legumes. Euphytica 2006, 147, 201-221.

5. Bani, M.; Rubiales, D.; Rispail, N. A detailed evaluation method to identify sources of quantitative resistance to Fusarium oxysporum f. sp. pisi race 2 within a Pisum spp. germplasm collection. Plant Pathol. 2012, 61, 532-542.

6. Becerra Lopez-Lavalle, L.A.; Gillespie, V.J.; Tate, W.A.; Ellis, M.H.; Stiller, W.N.; Llewellyn, D.L.; Wilson, I.W. Molecular mapping of a new source of Fusarium wilt resistance in tetraploid cotton (Gossypium hirsutum L.). Mol. Breeding 2012, 30, 1181-1191.

7. Oumouloud, A.; Arnedo-Andres, M.S.; Gonzalez-Torres, R.; Alvarez, J.M. Morphological and molecular characterization of melon accessions resistant to Fusarium wilts. Euphytica 2009, 169, 69-79.

8. Rispail, N.; Rubiales, D. Identification of sources of quantitative resistance to Fusarium oxysporum f. sp. medicaginis in Medicago truncatula. Plant Dis. 2014, 98, 667-673.

9. Vadivambal, R.; Jayas, D.S. Applications of thermal imaging in agriculture and food industry: A review. Food Bioprocess. Technol. 2011, 4, 186-199.

10. Chaerle, L.; Pineda, M.; Romero-Aranda, R.; Van Der Straeten, D.; Baron, M. Robotized thermal and chlorophyll fluorescence imaging of pepper mild mottle virus infection in Nicotiana benthamiana. Plant Cell Physiol. 2006, 47, 1323-1336. 
11. Zarco-Tejada, P.J.; Gonzalez-Dugo, V.; Berni, J.A.J. Fluorescence, temperature and narrow-band indices acquired from a UAV platform for water stress detection using a micro-hyperspectral imager and a thermal camera. Remote Sens. Environ. 2012, 117, 322-337.

12. Leinonen, I.; Jones, H.G. Combining thermal and visible imagery for estimating canopy temperature and identifying plant stress. J. Exp. Bot. 2004, 55, 1423-1431.

13. Calderon, R.; Navas-Cortes, J.A.; Lucena, C.; Zarco-Tejada, P.J. High-resolution air-borne hyperspectral and thermal imagery for early, detection of Verticillium wilt of olive using fluorescence, temperature and narrow-band spectral indices. Remote Sens. Environ. 2013, 139, 231-245.

14. Chaerle, L.; Hagenbeek, D.; de Bruyne, E.; Valcke, R.; van der Straeten, D. Thermal and chlorophyll-fluorescence imaging distinguish plant-pathogen interactions at an early stage. Plant Cell Physiol. 2004, 45, 887-896.

15. Stoll, M.; Schultz, H.R.; Baecker, G.; Berkelmann-Loehnertz, B. Early pathogen detection under different water status and the assessment of spray application in vineyards through the use of thermal imagery. Precis. Agric. 2008, 9, 407-417.

16. Wang, M.; Ling, N.; Dong, X.; Zhu, Y.; Shen, Q.; Guo, S. Thermographic visualization of leaf response in cucumber plants infected with the soil-borne pathogen Fusarium oxysporum f. sp. cucumerinum. Plant Physiol. Biochem. 2012, 61, 153-161.

17. Walter, A.; Studer, B.; Koelliker, R. Advanced phenotyping offers opportunities for improved breeding of forage and turf species. Ann. Bot. 2012, 110, 1271-1279.

18. Merlot, S.; Mustilli, A.C.; Genty, B.; North, H.; Lefebvre, V.; Sotta, B.; Vavasseur, A.; Giraudat, J. Use of infra-red thermal imaging to isolate Arabidopsis mutants defective in stomatal regulation. Plant J. 2002, 30, 601-609.

19. Raskin, I.; Ladyman, J.A.R. Isolation and characterization of a barley mutant with abscisic-acid-insensitive stomata. Planta 1988, 173, 73-78.

20. Jones, H.G.; Serraj, R.; Loveys, B.R.; Xiong, L.; Wheaton, A.; Price, A.H. Thermal infra-red imaging of crop canopies for the remote diagnosis and quantification of plant responses to water stress in the field. Func. Plant Biol. 2009, 36, 978-989.

21. Sirault, X.R.R.; James, R.A.; Furbank, R.T. A new screening method for osmotic component of salinity tolerance in cereals using infrared thermography. Func. Plant Biol. 2009, 36, 970-977.

22. Munns, R.; James, R.A.; Sirault, X.R.R.; Furbank, R.T.; Jones, H.G. New phenotyping methods for screening wheat and barley for beneficial responses to water deficit. J. Exp. Bot. 2010, 61, 3499-3507.

23. Zia, S.; Romano, G.; Spreer, W.; Sanchez, C.; Cairns, J.; Araus, J.L.; Mueller, J. Infra-red thermal imaging as a rapid tool for identifying water-stress tolerant maize genotypes of different phenology. J. Agron. Crop Sci. 2013, 199, 75-84.

24. Sepulcre-Canto, G.; Zarco-Tejada, P.J.; Jimenez-Munoz, J.C.; Sobrino, J.A.; Soriano, M.A.; Fereres, E.; Vega, V.; Pastor, M. Monitoring yield and fruit quality parameters in open-canopy tree crops under water stress. Implications for ASTER. Remote Sens. Environ. 2007, 107, 455-470.

25. Sanchez-Martin, J.; Mur, L.A.J.; Rubiales, D.; Prats, E. Targeting sources of drought tolerance within an Avena spp. collection through multivariate approaches. Planta 2012, 236, 1529-1545.

26. Oerke, E.C.; Steiner, U.; Dehne, H.W.; Lindenthal, M. Thermal imaging of cucumber leaves affected by downy mildew and environmental conditions. J. Exp. Bot. 2006, 57, 2121-2132. 
27. Tu, J.C.; Tan, C.S. Infrared thermometry for determination of root rot severity in beans. Phytopathology 1985, 75, 840-844.

28. Dong, X.; Ling, N.; Wang, M.; Shen, Q.; Guo, S. Fusaric acid is a crucial factor in the disturbance of leaf water imbalance in Fusarium-infected banana plants. Plant Physiol. Biochem. 2012, 60, 171-179.

29. Wu, H.-S.; Bao, W.; Liu, D.-Y.; Ling, N.; Ying, R.-R.; Raza, W.; Shen, Q.-R. Effect of fusaric acid on biomass and photosynthesis of watermelon seedling leaves. Caryologia 2008, 61, 258-268.

30. Mepsted, R.; Flood, J.; Cooper, R.M. Fusarium wilt of oil palm II. Stunting as a mechanism to reduce water stress. Physiol. Mol. Plant Pathol. 1995, 46, 373-387.

31. Wang, M.; Xiong, Y.; Ling, N.; Feng, X.; Zhong, Z.; Shen, Q.; Guo, S. Detection of the dynamic response of cucumber leaves to fusaric acid using thermal imaging. Plant Physiol. Biochem. 2013, 66, 68-76.

32. Bani, M.; Rispail, N.; Evidente, A.; Rubiales, D.; Cirnmino, A. Identification of the main toxins isolated from Fusarium oxysporum f. sp. pisi race 2 and their relation with isolates' pathogenicity. J. Agric. Food Chem. 2014, 62, 2574-2580.

33. Sarhan, A.R.T.; Hegazi, A.M.A. Effect of nitrogen, phosphorus and potassium on sensitivity of pepper plants to Fusarium wilt toxin. Acta Phytopathol. Entomol. Hung. 1988, 23, 57-60.

34. Rispail, N.; Bani, M.; Rubiales, D. Resistance reaction of Medicago truncatula genotypes to Fusarium oxysporum: Effect of plant age, substrate and inoculation method. Crop Past. Sci. 2015, in press.

35. Bauriegel, E.; Giebel, A.; Geyer, M.; Schmidt, U.; Herppich, W.B. Early detection of Fusarium infection in wheat using hyper-spectral imaging. Comput. Electon. Agric. 2011, 75, 304-312.

36. Prats, E.; Gay, A.P.; Mur, L.A.J.; Thomas, B.J.; Carver, T.L.W. Stomatal lock-open, a consequence of epidermal cell death, follows transient suppression of stomatal opening in barley attacked by Blumeria graminis. J. Exp. Bot. 2006, 57, 2211-2226.

37. Mur, L.A.J.; Simpson, C.; Gay, A.; Smith, J.A.; Paveley, N.; Sanchez-Martin, J.; Prats, E. Stomatal lock-up following pathogenic challenge: Source or symptom of costs of resistance in crops? Plant Pathol. 2013, 62, 72-82.

(C) 2015 by the authors; licensee MDPI, Basel, Switzerland. This article is an open access article distributed under the terms and conditions of the Creative Commons Attribution license (http://creativecommons.org/licenses/by/4.0/). 